\title{
VAPD-2D: Simulador pa- ra Apoio ao Ensino de Engenharia Ambiental
}

\section{Carlos Vitor de Alencar Carvalho Docente do Programa de Mestrado \\ Profissional em Educação Matemáti-} ca

Curso de Sistemas de Informação e Matemática

Universidade Severino Sombra -

Vassouras - RJ

e-mail: cvitorc@gmail.com
Janaina Veiga Carvalho

Docente do Programa de Mestrado

Profissional em Educação Matemática

Curso de Sistemas de Informação e Matemática

Universidade Severino Sombra - Vassouras - RJ e-mail:

janainavcarvalho@gmail.com
Júlio César da Silva

Docente do Curso de Ciência da Computação

UNIFESO - Centro Universitário da Serra dos Órgãos - Teresópolis - RJ

Pesquisador do DEC/PUC-Rio -

Pontifícia Universidade Católica do

Rio de Janeiro - Rio de Janeiro - RJ

e-mail: jcesarop@gmail.com

Resumo Este trabalho apresenta o desenvolvimento de um software educativo, chamado VAPD-2D para apoio às soluções para problemas da Engenharia Ambiental, mais especificamente de problemas onde o transporte por difusão é predominante. O mecanismo de transporte por difusão tem várias aplicações práticas na Engenharia, destacando os estudos da contaminação do solo e de águas subterrâneas por produtos químicos orgânicos e inorgânicos. Estes estudos envolvem, basicamente, a determinação dos parâmetros físicos e químicos dos contaminantes e a forma como estes se movem, quando dissolvidos na água subterrânea. O VAPD-2D é um simulador numérico de interface bastante simples que permite aos estudantes da Engenharia simular $e$ testar parâmetros para diversos tipos de problemas.

Palavras-Chave: software educativo, simulações numéricas, equação da difusão, método das diferenças finitas

\begin{abstract}
This work describes the development of an educational, called VAPD-2D for support to the teaching of problems of the Environmental Engineering, more specifically of problems where the transport for diffusion is predominant. The transport mechanism for diffusion has several practical applications in the Engineering, highlighting the studies of the contamination of the soil and of underground waters for organic and inorganic chemical products. These studies involve, basically, the determination of the physical and chemical parameters of the contaminants and the form that they move, when dissolved in the groundwater. The VAPD-2D is a numerical simulator of quite simple interface that allows to the students of the Engineering to simulate and to test parameters for varied types of problems.
\end{abstract}

Keywords: educational software, numerical simulation, diffusion equation, finite differences methods. 


\section{Introdução}

Os debates sobre e a utilização e o desenvolvimento de novas Tecnologias na educação, vêm aumentando ultimamente. As Tecnologias estão inseridas em todas as atividades do dia a dia das pessoas e é um direito do estudante usufruir de uma educação que incluía até mesmo uma "alfabetização tecnológica" como cita Borba [2]:

"(...) nesse sentido, a discussão sobre a informática na educação (...) deve ser vista como um direito e, portanto, nas escolas públicas e particulares o estudante deve poder usufruir uma educação que no momento atual inclua, no mínimo, uma alfabetização tecnológica" [2].

Tecnologia pode ser uma grande aliada para ajudar no pensar de um método pedagógico que consista em atividades atraentes para o ensino e uma das formas de utilização desta Tecnologia é através de softwares educacionais. Eles medeiam a prática pedagógica oferecendo desafios importantes voltados à produção intelectual, em que o aluno passa a ser produtor de conhecimento e a ação docente dá lugar à relação dialógica que permite ao professor e ao aluno aprender a aprender, num processo coletivo para a produção do conhecimento [11].

A utilização de softwares para apoio ao ensino de Engenharia também se tornou um grande desafio e é uma perspectiva que não pode mais ser ignorada pelas Instituições de Ensino e professores. Algumas das principais vantagens da sua utilização como apoio ao ensino se deve principalmente seu grande potencial pedagógico e vários benefícios como, aumentar a capacidade cognitiva e aproximar ainda mais a informação dos alunos [10].
No caso da Engenharia Ambiental, a construção de softwares é de grande importância para entendimento dos mecanismos de transporte de contaminantes. Através destes softwares pode-se possibilitar aos alunos uma análise de sensibilidade dos parâmetros envolvidos nos processos, através da manipulação destes parâmetros via interface gráfica do software construído.

\section{Simuladores para Engenharia}

No Ensino de Engenharia é uma prática comum a utilização de simuladores para exemplificação de um determinado assunto. $\mathrm{O}$ ensino apoiado em simuladores facilita a compreensão dos alunos por determinado tópico apresentado, como pode ser visto em Guillermo [8] que apresenta o poder de simuladores na área de Hidráulica.

Como são vários os exemplos de simuladores numéricos desenvolvidos para apoio ao ensino da Engenharia, neste trabalho serão apresentados alguns simuladores construídos pelos autores deste artigo. Em Carvalho [4], pode-se verificar um simulador para estudo do comportamento da ventilação cruzada em ambientes construídos (Figura 1). Em Carvalho [3] é abordado outro exemplo sobre estudo de tensões através do Círculo de Mohr (Figura 2).

A implantação de um sistema de controle como um programa de objetos concorrentes é baseado em modelos de concorrência, o que permite a representação do sistema como uma rede de elementos cooperativos, neste caso os Agentes. O uso da mesma classe base para a derivação de todas as classes garante a uniformidade da interface para todos os elementos, facilitando a programação e minimizando erros de implementação.

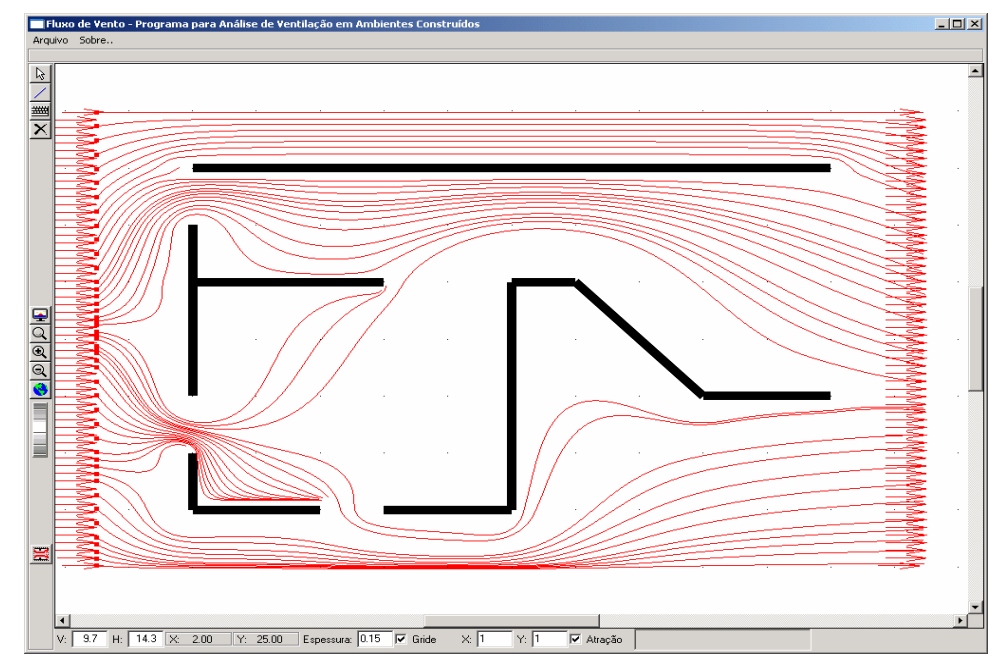

Figura 1 - FLUXOVENTO - Simulador para estudo de ventilação cruzada em ambientes construídos. 


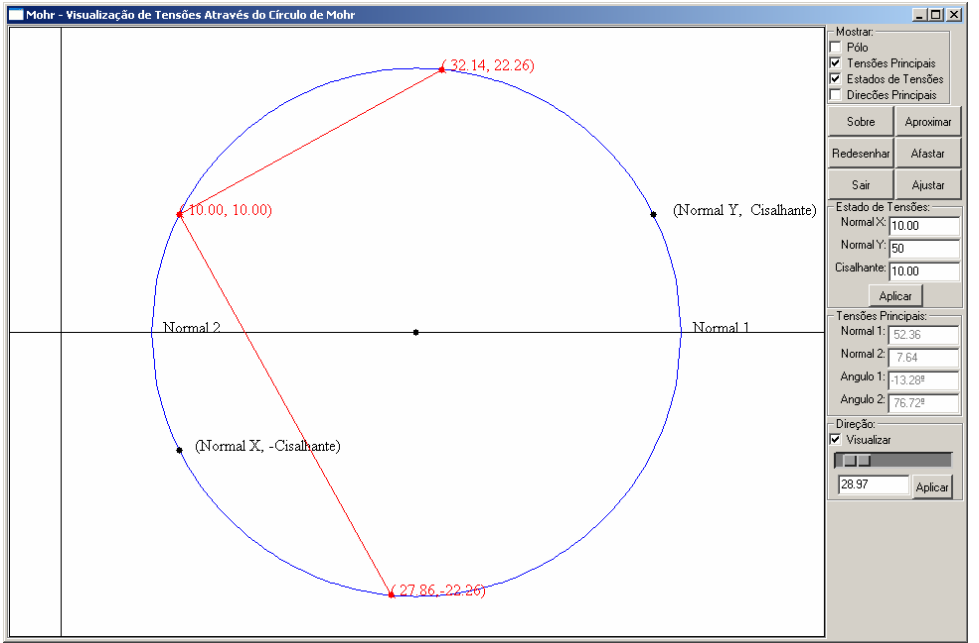

Figura 2 - CIRCULOMOHR - Simulador para estudo de tensões planas através do Círculo de Mohr.

\section{Desenvolvimento computacional}

O software VAPD-2D tem como base o conceito matemático da equação da difusão em duas dimensões - 2D [7] e técnicas de computação gráfica [6]. A equação da difusão é um dos mecanismos de transportes de partículas e indica como uma determinada concentração c se espalha em uma região, em função de parâmetros, conhecidos como coeficientes difusão definidos pelo usuário. Os outros mecanismos, que não são objetivos de estudo deste artigo são: dispersão e advecção [1].

Este mecanismo de transporte tem várias aplicações práticas na Engenharia, destacando: estudos da contaminação do solo e de águas subterrâneas por produtos químicos orgânicos e inorgânicos. Estes estudos envolvem, basicamente, a determinação dos parâmetros físicos e químicos dos compostos (contaminantes), bem como a forma pelos quais estes se movem, quando dissolvidos, na água subterrânea.

A equação (1) mostra a equação da difusão em uma dimensão.

$$
\frac{\partial c}{\partial t}=k \frac{\partial^{2} c}{\partial x^{2}}
$$

A equação de difusão em duas dimensões é definida por:

$$
\frac{\partial c}{\partial t}=k_{x} \frac{\partial^{2} c}{\partial x^{2}}+k_{y} \frac{\partial^{2} c}{\partial y^{2}}
$$

onde:

$\frac{\partial c}{\partial t}$ Indica a variação da concentração com o tempo;

$k_{x} e k_{y}$ Indicam os coeficientes de difusão nas direções $\mathrm{x}$ e y respectivamente.

A Figura 3 mostra graficamente o efeito da equação ao longo do tempo.

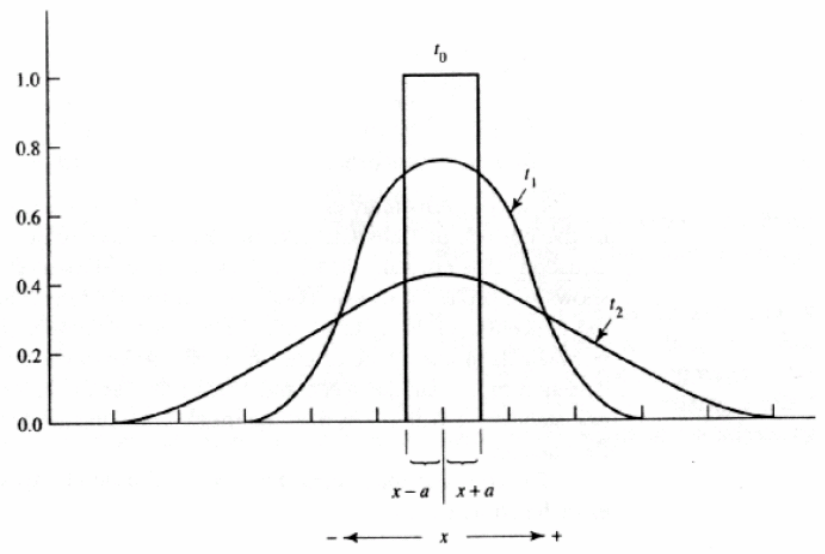

Figura 3 - Efeito do mecanismo de difusão: variação da concentração ao longo do tempo [1]. 


\subsection{Solução Numérica}

Neste trabalho foi utilizado o Método das Diferenças Finitas (MDF) [5] para discretizar a equação 2. O MDF foi um dos primeiros métodos numéricos desenvolvidos, sendo aplicado, até na atualidade, a uma extensa gama de problemas. Neste método, utiliza-se uma malha, grid, sobre todo o domínio físico do problema (Figura 4 e Figura 5), a qual contém determinados pontos onde são efetuadas as aproximações envolvidas. Representações das derivadas em diferenças finitas são baseadas na expansão em série de Taylor.
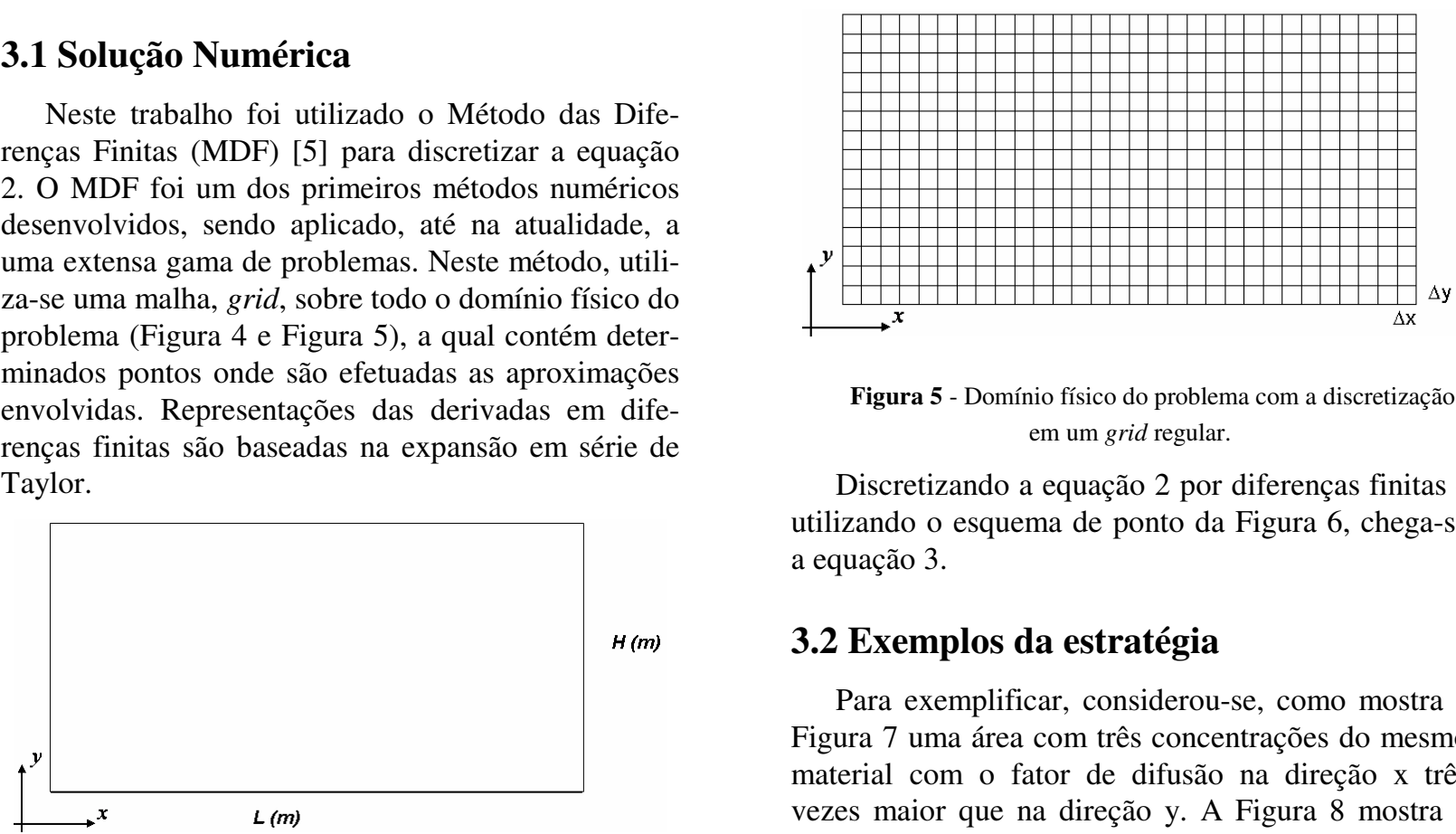

Figura 5 - Domínio físico do problema com a discretização em um grid regular.

Discretizando a equação 2 por diferenças finitas e utilizando o esquema de ponto da Figura 6, chega-se a equação 3 .

\subsection{Exemplos da estratégia}

Para exemplificar, considerou-se, como mostra a Figura 7 uma área com três concentrações do mesmo material com o fator de difusão na direção $\mathrm{x}$ três vezes maior que na direção y. A Figura 8 mostra a distribuição do material após a simulação.

Figura 4 - Domínio físico do problema.

$$
\frac{\left(c_{t+1, i, j}-c_{t, i, j}\right)}{\Delta t}=k_{x} \frac{\left(c_{t, i, j+1}+c_{t, i, j-1}-2 c_{t, i, j}\right)}{\Delta x^{2}}+k_{y} \frac{\left(c_{t, i+1, j}+c_{t, i-1, j}-2_{t, i, j}\right)}{\Delta y^{2}}
$$

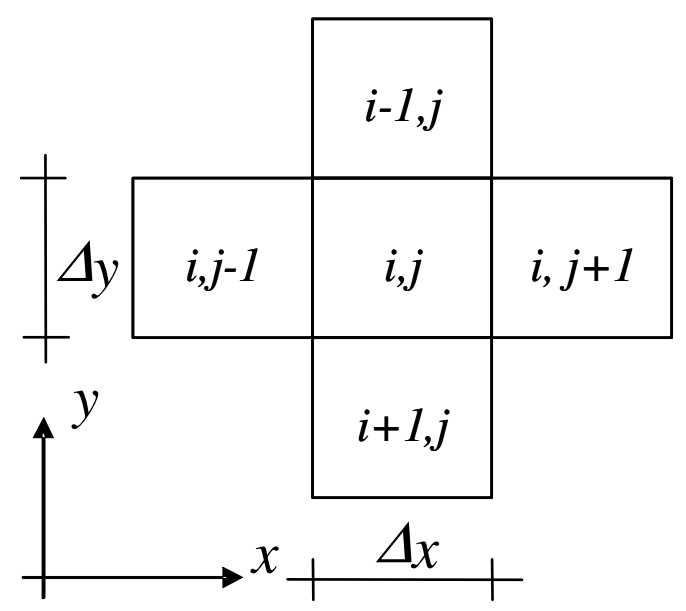

Figura 6 - Esquema dos pontos utilizados para discretização da equação 2. 


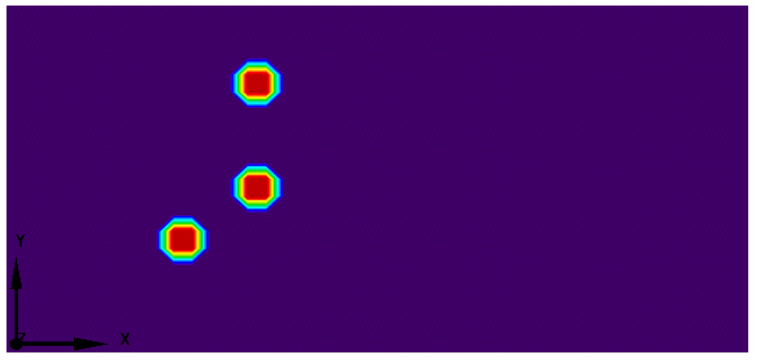

Figura 7 - Modelo com a configuração inicial das concentrações.

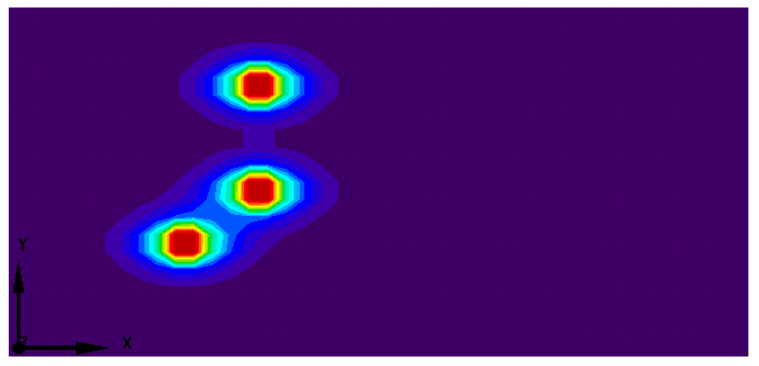

Figura 8 - Modelo com a configuração final das concentrações.

\subsection{A interface gráfica do VAPD-2D}

A metodologia descrita anteriormente é o núcleo central do software VAPD-2D. O sistema possui interface bastante simples que permite ao aluno definir concentrações e estudar e analisar sua variação com o tempo (em segundos) na área a ser modelada. Desenvolvido para alunos que estão no $4^{\circ}$ ano dos cursos de Engenharia Ambiental, o VAPD-2D será de grande valia para estudos de problemas teóricos e práticos da área. O software foi desenvolvido para ter uma grande iteratividade, no que diz respeito à definição de todos os parâmetros de concentração e coeficientes de difusão além da definição de regiões onde não existe variação de concentração.

Este software foi desenvolvido usando as linguagens $\mathrm{C} / \mathrm{C}++$, utilizando a biblioteca gráfica de interface IUP [9] e o sistema gráfico OpenGL [12]. A Figura 9 mostra a interface do VAPD-2D ressaltando algumas das suas principais funcionalidades.

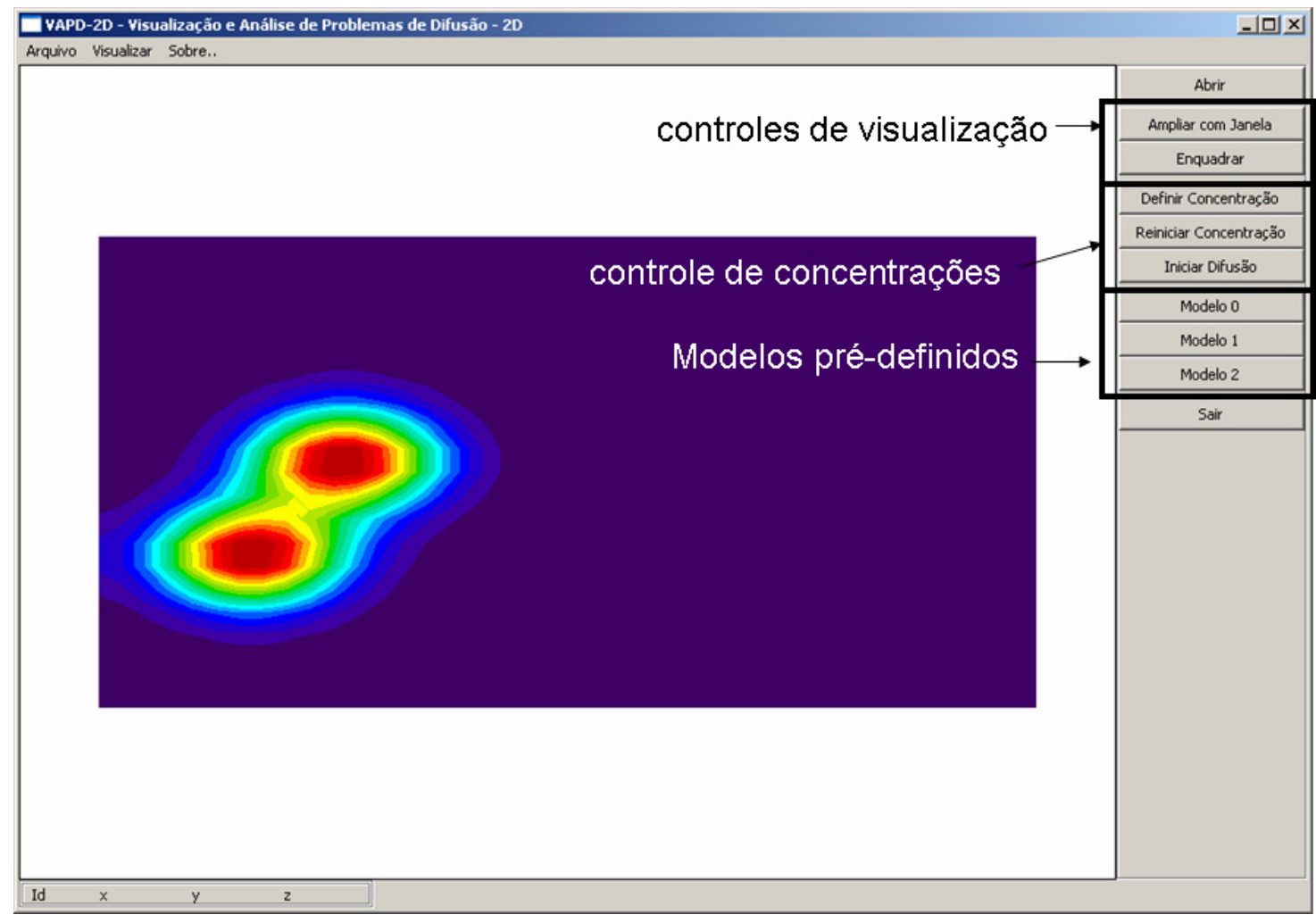

Figura 9 - Interface do Sistema VAPD-2D.

\section{Aplicações Educacionais}

No Curso de Engenharia Ambiental da Universidade Severino Sombra (Vassouras/RJ), foi incluída na sua matriz curricular uma disciplina chamada "Modelagem Computacional na Engenharia Ambien- tal". Nesta disciplina é dada ênfase na importância da Modelagem Computacional, bem com as suas condições em que devem ser utilizadas no seu dia a dia. $\mathrm{O}$ VAPD-2D será de grande valia em disciplinas com este foco. Em seguida são mostradas algumas aplicações que podem ser desenvolvidas em sala de aula 
Revista Eletrônica TECCEN - ano I - volume 2 - Out-Dez de 2008 ISSN 1984-0993

pelo professor junto com os alunos.

Aplicação 1: Considere um depósito com material contaminante que possui um vazamento como mostra a Figura 10. Utilize o VAPD-2D para verificar se o vazamento atingirá o nível de água subterrâ- nea. A Figura 11 e a Figura 12 mostram, respectivamente, os dados de entrada e a configuração inicial e final da concentração.

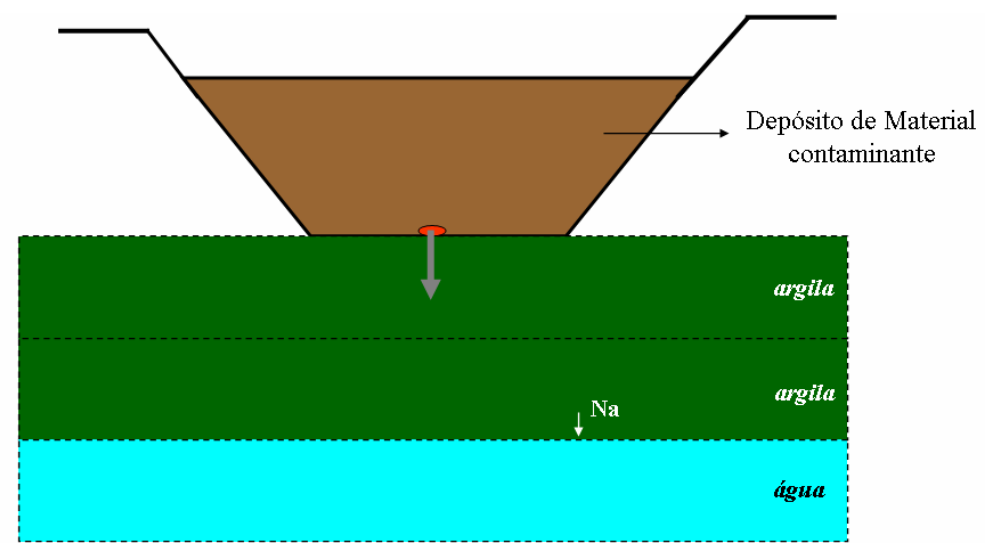

Figura 10 - Depósito com material contaminante.

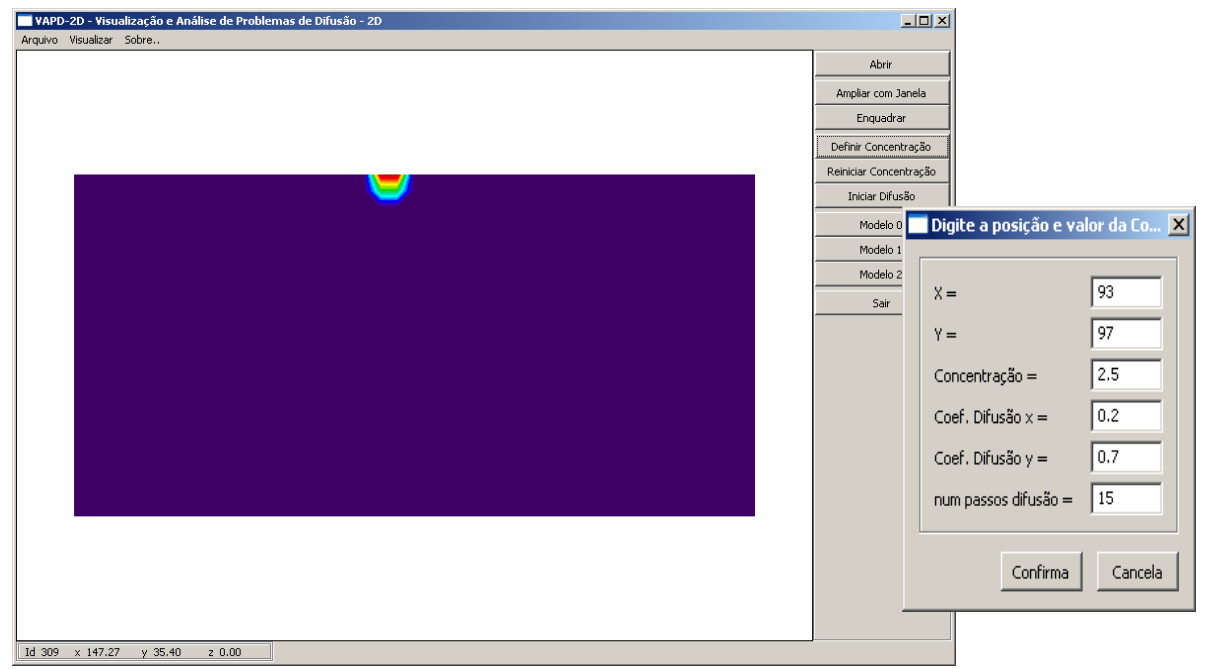

Figura 11 - Configuração inicial da concentração do exemplo da Figura 10.

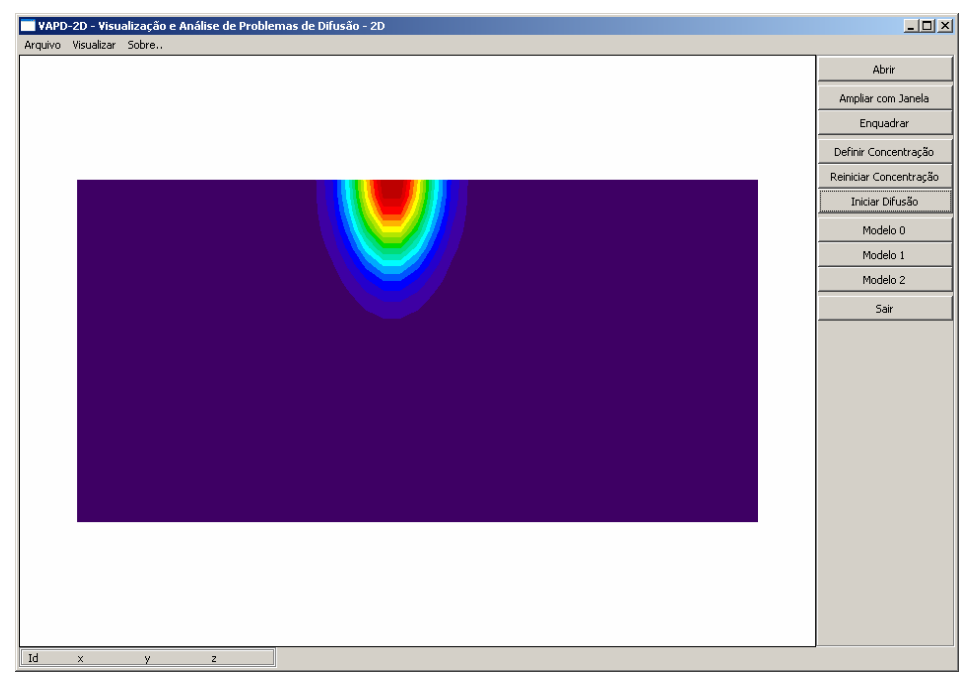

Figura 12 - Configuração final da concentração do exemplo da Figura 10. 
Aplicação 2: Considere um material que será conduzido através de um canal ocorrendo na saída do mesmo o fenômeno de dispersão. Utilize o VAPD-2D para verificar a configuração final após o material sair do canal. A Figura 13 mostra a configuração inicial e a Figura 14 mostra a configuração após a simulação.

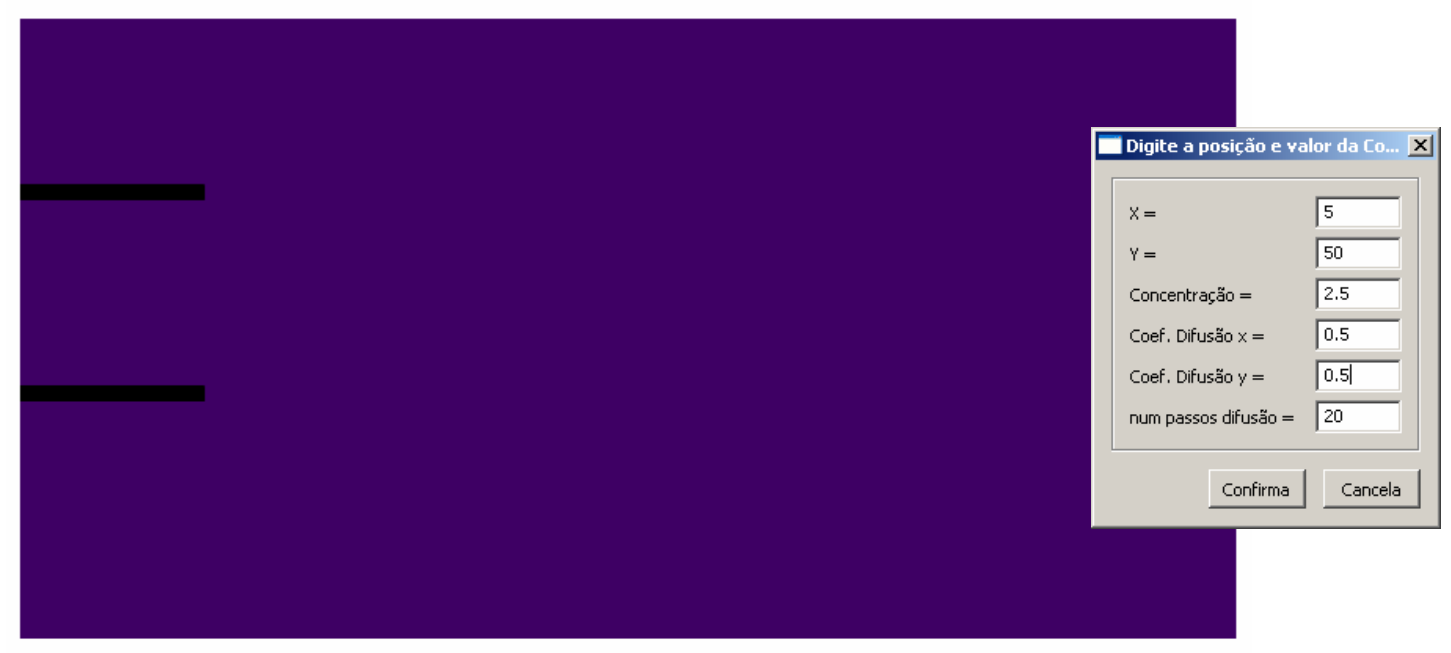

Figura 13 - Configuração inicial com a visualização do canal.

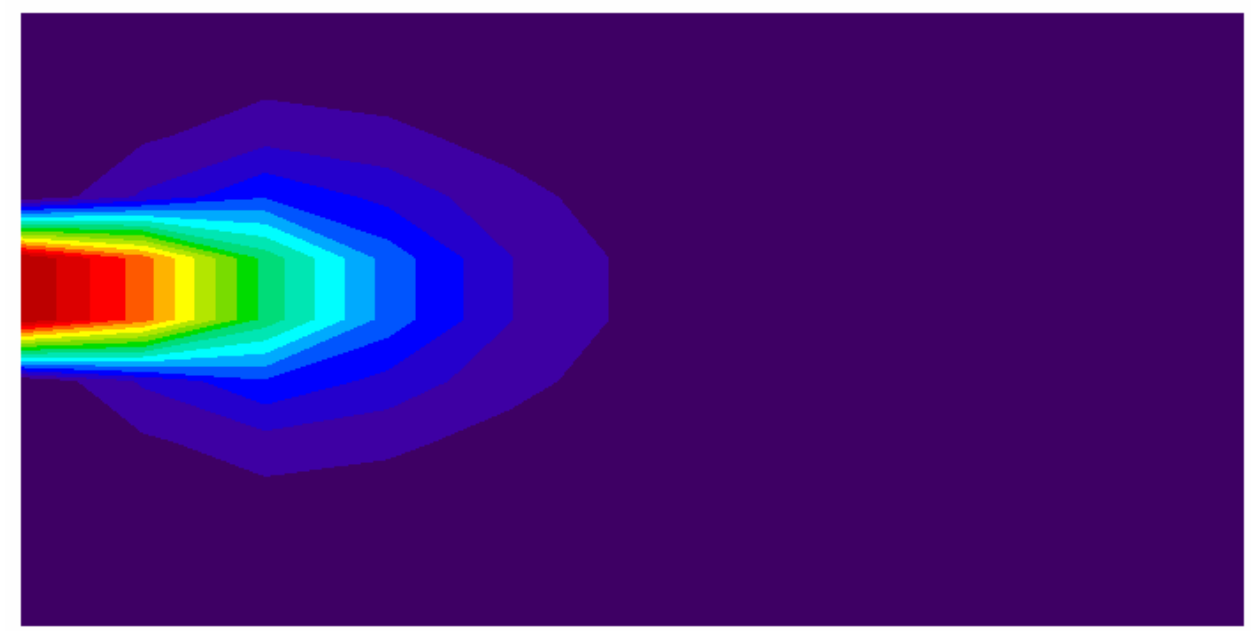

Figura 14 - Configuração final após a simulação.

\section{Considerações Finais}

A utilização de softwares educacionais, como no caso do VAPD-2D, permitem ao professor e aos alunos fazerem observações e estudos com várias definições em um curso espaço de tempo. Espera-se então, com este software aumentar o conteúdo assimilado pelos alunos e tornar as aulas mais dinâmicas. É importante ressaltar que o uso de softwares educacionais não deve ser ignorado pelas Instituições de Ensino devido o seu grande potencial pedagógico.

Este trabalho é mais uma contribuição para a reflexão sobre as relações que se estabelecem entre a Tecnologia e a Educação, buscando ressignificar as práticas pedagógicas cotidianas à luz dos novos avan- ços das tecnologias da informação. A próxima etapa desta pesquisa será utilizar o software com alunos do público alvo para verificar sua avaliação/validação em sala de aula.

\section{Agradecimentos}

Os autores Carlos Vitor de Alencar Carvalho e Janaina Veiga Carvalho agradecem à FAPERJ (Fundação de Amparo à Pesquisa do Estado do Rio de Janeiro) pelo auxílio financeiro recebido e a FUNDADESP (Fundação Nacional de Desenvolvimento do Ensino Superior Particular) pela bolsa de produtividade de pesquisa de doutorado. 


\section{Referências}

[1] I. .T. ARAÚJO, de Aplicações de Métodos de Análise Numérica de Transporte Reativo de Multicompostos em Meios Porosos. Dissertação de Mestrado, PUC-Rio, 2001.

[2] Marcelo C. BORBA e M. G. PENTEADO Informática e Educação Matemática - coleção tendências em Educação Matemática - Autêntica, Belo Horizonte - 2007

[3] C. V. A. CARVALHO, J. V. CARVALHO Um software educacional gráfico e interativo para apoio ao estudo de tensões planas através do círculo de Mohr. Revista de Informática Mater $D e i$, v. 4, p. 1-12, 2008.

[4] C. V. A. CARVALHO, , L. F. MARTHA, , W. TEIXEIRA - FLUXOVENTO - Um simulador gráfico interativo para o estudo de ventilação em ambientes construídos. In: VII Encontro Nacional sobre Conforto no Ambiente Construído (ENCAC), 2005, Maceió. IV Encontro LatinoAmericano sobre Conforto no Ambiente Construído e VIII Encontro Nacional sobre Conforto no Ambiente Construído, Maceió; v. 1. p. 350360, 2005.

[5] C. CUNHA, Métodos Numéricos, Editora Unicamp, 2003.

[6] J. D. FOLEY, Computer Graphics - Principles And Practice - Addison-Wesley Publishing Company, 1990.

[7] A. O. FORTUNA Técnicas Computacionais para Dinâmica dos Fluidos, Editora Edusp, 2000.

[8] O. E. P. GUILLERMO, L. M. R. TAROUCO, , L. A. M. ENDRES. O Poder das Simulações no ensino de hidráulica. RENOTE. Revista Novas Tecnologias na Educação, Porto Alegre - Brasil; v. 3, n. 1, 2005.

[9] C. H. LEVY, L. H. FIGUEIREDO, M. GATTASS, C. LUCENA and D. COWAN IUP/LED: A Portable User Interface Development Tool. Software: Practice \& Experience, 26 \#7, pp. 737-762, 1996.

[10] J. L. de S. MENDES, C. V. A. CARVALHO, J. V. CARVALHO, CONSTRUFIG3D: Uma Ferramenta Computacional para apoio ao ensino da Geometria Plana e Espacial. RENOTE. Revista Novas Tecnologias na Educação, v. 5, n 1, p. 1/10-10, 2007.

[11] A. M. S. PAIVA, J. V. CARVALHO, C. V. A. CARVALHO, I da P. G. PORTO, A integração da TIC na Escola Básica: Questões para Avaliação, Revista Eletrônica TECCEN, ano 1, volu- me 1, 2008.

[12] M. WOO, N. JACKIE et al, OpenGL Programming Guide, Third Edition (OpenGL, Version 1.2), Addison-Welsey, 1999. 